Research Journal of Applied Sciences, Engineering and Technology 6(15): 2706-2710, 2013

DOI:10.19026/rjaset.6.3774

ISSN: 2040-7459; e-ISSN: 2040-7467

(C) 2013 Maxwell Scientific Publication Corp.

Submitted: September 26, 2012

Accepted: December 13, 2012

Published: August 20, 2013

\title{
Research Article \\ Developments in Researches on the Disasters during Cavern Construction in Salt Beds with Multi-Interlayer
}

\author{
Huafu Qiu, Deyi Jiang, Jie Chen, Song Ren and Lin Li \\ State Key Laboratory for the Coal Mine Disaster Dynamics and Controls, Chongqing \\ University, Chongqing 400044, China
}

\begin{abstract}
This study analyses the developments in researches on the disasters during cavern construction in salt beds with multi-interlayer. Relative to the foreign large salt dome landscape, there are many layers of mudstone in salt beds which has a thin single layer. And such special geological conditions increase the difficulty of solution mining in the control of cavity shape and stability of the cavity, which easily leads to disasters. To statistical analysis the disasters in process of cavern building with multi-interlayer in china, it show that: taking Chinese geological conditions for example, the disasters during cavern construction in salt beds with multi-interlayer caused the failure of cavity can be divided into three categories: geological conditions in beds; the damage of cavity wall in the process of cavity building; the distortion of cavity shape expansion. To analyze predisposing factors lead to salt cavern storage failure in detail, the mechanism of the corresponding factors lead to disaster has been summarized. Finally, some suggestions has been proposed to avoid the disaster maybe happened.
\end{abstract}

Keywords: Multi-interlayer, the shape deformity, the interlayer collapse, the surface subsidence, washing techniques

\section{INTRODUCTION}

Rock salt is a kind of soft rock and it has excellent physical and mechanical properties of the low permeability and good self-healing ability which make it to be widely recognized as the ideal energy underground storage sites. The underground energy storage of Salt rock has been built and accomplished successfully widely in many countries. A washing technique has been developed to form a spherical cavity in massive salt. Foreign researchers have studied on the mechanism of flow and controlled dissolution of salt in solution mining, the solubility properties of rock salt and the shape control techniques of cavities in salt formation for underground gas storage. To numerical analysis, the influencing factors in salt formation containing the cavity shape, size, depth and mechanical parameters have been accomplished, so was the optimal design parameters of the cavity for safe operation of gas storage in salt rock . Relative to the foreign large salt dome landscape, there are many layers of mudstone in salt beds which has a thin single layer. And such special geological conditions increase the difficulty of solution mining in the control of cavity shape and stability of the cavity, making caverns irregular shape and poor mechanical properties, which is less than the expectations of the desired effect. The collapse of the interlayer may damage the casing of the cave and influence the shape of the cavity, which is a trouble for cavity building. Based on these specific circumstances, the domestic scholars studied on the physical and mechanical properties of the layered rock salt and got a systematic study of mechanical properties and solubility characteristics of rock salt in different conditions; and proposed several damage model of interlayer collapse; the preliminary studies on the influence about mudstone interlayer to cavity stability also be carried out. Kazemi and Jessen (1964) study the mechanism of flow and controlled dissolution of salt in solution mining. Tao and Ma (2009) have a research of the research of salt cavern 3D modeling technology. Ehgartner and Park (2009) study the sensitivity of storage field performance to geologic and cavern design parameters in salt domes. Fuenkajorn (2010) analyse the Prediction of cavern configurations from subsidence data. Jiang et al. (2009) study experimental research on acoustic and dissolved properties of stress damaged salt rock. Liang et al. (2010) have a research of test study of strain rate effects on mechanical performances of salt rock.

For the Energy storage construction, we are very concerned about the shape of the cavity. During the construction process of underground energy storage, the concentration distribution is the key factor affecting the shape of the cavity, which impacts the dissolution rate of the rock salt. But there is no systematic existing study on the concentration field and its variation, particularly

Corresponding Author: Huafu Qiu, State Key Laboratory for the Coal Mine Disaster Dynamics and Controls, Chongqing University, Chongqing 400044, China

This work is licensed under a Creative Commons Attribution 4.0 International License (URL: http://creativecommons.org/licenses/by/4.0/). 
the effects of the freshwater flow, distance between wash pipes and circulation method. The researches on the distribution of concentration field are useful for the shape control and stability in cavity built.

This study analyses the developments in researches on the disasters during cavern construction in salt beds with multi-interlayer. Relative to the foreign large salt dome landscape, there are many layers of mudstone in salt beds which has a thin single layer. And such special geological conditions increase the difficulty of solution mining in the control of cavity shape and stability of the cavity, which easily leads to disasters. To statistical analysis the disasters in process of cavern building with multi-interlayer in china, it show that: taking Chinese geological conditions for example, the disasters during cavern construction in salt beds with multi-interlayer caused the failure of cavity can be divided into three categories: geological conditions in beds; the damage of cavity wall in the process of cavity building; the distortion of cavity shape expansion. To analyze predisposing factors lead to salt cavern storage failure in detail, the mechanism of the corresponding factors lead to disaster has been summarized. Finally, some suggestions has been proposed to avoid the disaster maybe happened.

\section{THE KEY DISASTER DURING THE CAVITY BUILDING OF SALT ROCK}

Rock salt gas storage: First, select the location for drilling and drilling its, place casing to be the position where is water-soluble salt layer of the cave, the platform was formed based on the basic principles of the chamber building in water-soluble; Second, freshwater enters into layer cavity through the annulus and is discharged through the smallest tubing string. We control dissolution upward by injecting oil to the designed position for pad when the diameter of cavity expand to the designed size which through the continuous dissolution of the wall; Finally, through controlling the oil pad position and change the water injection flow and the casing spatial position to control the shape of the cavity expansion and ultimately achieve the purpose of the cave. This cavity building process is as shown in Fig. 1.

However, during the cavity building, the expansion process of cavity is invisible and the factors as the underground salt rock quality and insoluble mudstone, led to the failure of the cavity building, which cause secondary disasters such as the shape deformity of cavity, the cavity instability and surface subsidence and other disasters. So critical to disaster factors in a salt cavern construction process can greatly reduce the occurrence of secondary disasters of the cave. By cavity-building achievements of statistics around the world, the disaster factors in cavity building: the shape deformity of the cavity deformity, mezzanine collapsed,

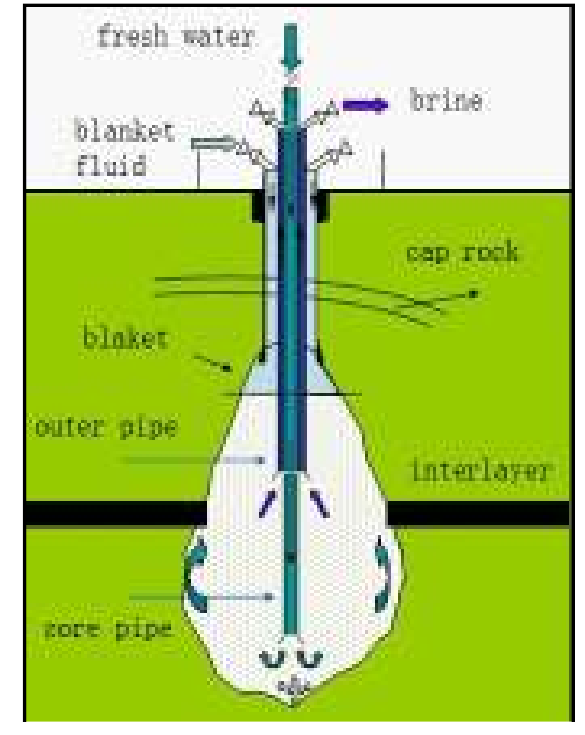

Fig. 1: The process of cavity building

weakening the rock strength by brine, casing breakage and surface subsidence.

The shape deformity of the cavity: The shape deformity of the cavity is the most important factor in cavity building, which is the most common type of disaster shown in Fig. 2. To avoid the cavity instability and failure caused by the unreasonable shape of cavity, we must make out the shape design of cavity before the cavity building. To analysis the mechanical properties of the salt rock by the uniaxial and triaxial compression test, especially the creep properties, Karl Heinz Lux researched the shape design of cavity. Based on the research of the influence that the rock mechanical stability to the geometry of the cavity and ensuring the stability, Qu Ping has proposed the optimal design of the cavity shape which solve the geometric parameters of the upper melting chamber wall using the pressure arch model, solve the parameters of the lower melting parietal using the most dangerous sliding surface model and analyzed the influence of internal pressure on the parietal stability, the minimum internal pressure determines the upper melting of the parietal design, the maximum internal pressure determines the lower part of the dissolved parietal design. Pre-design of the proposed shape of the cavity before the cavity building is an important means to avoid the failure of the cavity, so statistical analysis of key factors causing the shape deformity of the cavity must be made. According to previous studies, we found that factors causing the shape deformity of the cavity: the taste of salt, the salt layer distribution, cavity building process.

The influence to the shape of the cavity by the salt taste: The degree of salt taste determines the dissolution 

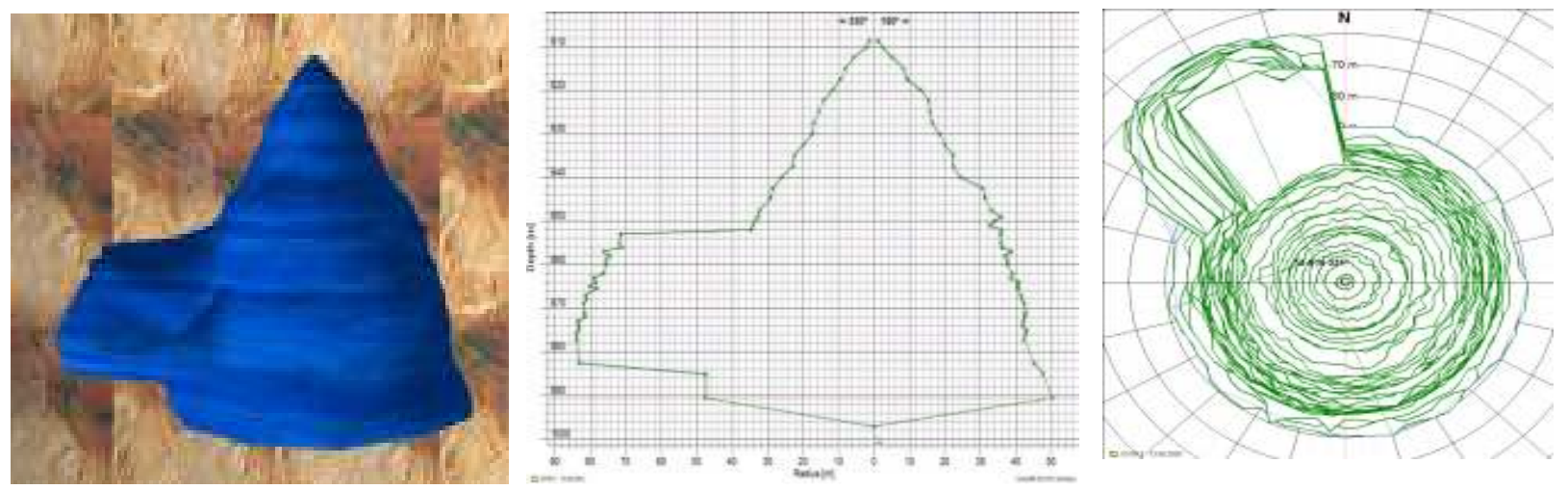



(Perspective view)

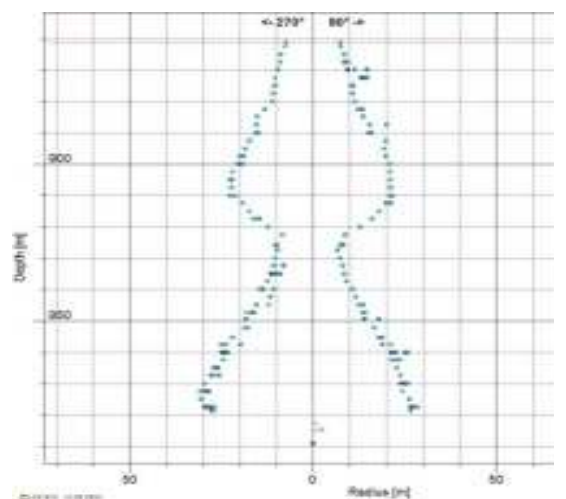

(Cross-sectional view)

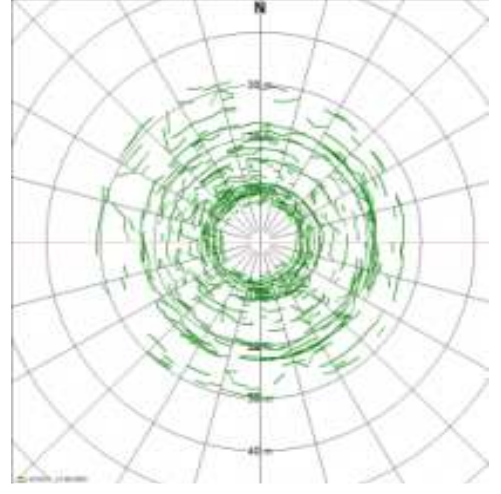

(Top view)

Fig. 2: The shape deformity of cavity

rate of the rock salt which influences the pace of expansion of the cavity shape and the finial cavity shape. Generally, the greater surface of the water and mineral salts, the faster the salt dissolution. Hans Ulrich Rohr (1979) gives the dissolution velocity curve of different salts, it shows that the $\mathrm{MgCl}_{2} \cdot 6 \mathrm{H}_{2} \mathrm{O}$ dissolve fastest, the maximum dissolution rate of $1.037 \times 10^{-3} \mathrm{~cm}$ / $\mathrm{s}$, the melting speed of $\mathrm{KCl}$ at low concentrations is higher than that of $\mathrm{NaCl}$, but in high concentrations of dissolved speed lower than $\mathrm{NaCl}$, while $\mathrm{Na}_{2} \mathrm{SO}_{4}$ $10 \mathrm{H}_{2} \mathrm{O}, \mathrm{MgSO}_{4} \cdot \mathrm{H}_{2} \mathrm{O}$ and $\mathrm{K}_{2} \mathrm{SO}_{4}$ dissolution rate is lower than that of $\mathrm{NaCl}$. To study the rock salt and the rock salt with mud dissolution test under room temperature and high temperature, Ma Honglin found the relationship between density and time showing exponential decay and its parameters were fitted and come to that the dissolution rate of pure rock salt is 1.26 times than the rock salt with mud under room temperature, the dissolution rate of pure rock salt at the high temperature of $86^{\circ} \mathrm{C}$ and high temperature of 50 ${ }^{\circ} \mathrm{C}$, respectively is 2.32 and 1.49 times than the rock salt with mud under room temperature. The cavity form varies under different tastes of salt. It is benefit for the shape control of cavity to choose the salt beds which has great taste for cavity building.

The influence to the shape of the cavity by the distribution of salt layer: The distribution of salt layer refers to the distribution of the salt layer in the strata, mainly divided into the salt dome type and the bedded salt type. The salt dome type is only a layer with large thickness, usually ranging from a few hundred meters to thousand meters which is useful for cavity building for its high salt taste and uniform distribution, under the process of standard-made cavity, the shape of the cavity could meet the design requirements, cavity instability disasters rarely occur. Bedded salt rock is salt layers inter-bedded with mudstone. The salt layer thickness does not exceed $200 \mathrm{~m}$, interlayer thickness between $0.5-5 \mathrm{~m}$ and with many layers. Durie overlay the same thickness salt tablets and the insoluble interlayer and then made the dissolution test in 1964 . He found that the existence of the interlayer accelerate the dissolution of rock salt, the same thickness samples dissolution with same rate which has not to do with the thickness of the interlayer. The thinner the salt layers, the faster the dissolution. In short, the existence of the interlayer is the key which influence the continuous of the cavity shape, unfavorable cavity shape control, resulting in failure of the cave and even the overall instability of the cavity.

The influence to the shape of the cavity by washing techniques: The washing technique is a way to adjust the injection brine flow and casing spatial position to control the dissolution rate of the salt cavern wall, to 
achieve the purpose of controlling the shape of the cavity. Taking the project of energy reservoir in Jin-tan for basis, Wan Yu-Jin has conducted the study on the shape control techniques in cavity building, the study shows the parameters influence the shape of cavity includes the location of casing pipes, the method and location of oil pad, the circle method and the water flow, the nice way to control cavity shape need to optimize the parameters and adjust the time.

The interlayer collapse: In the interlayer-containing salt cavity construction process, due to the mezzanine mostly insoluble matter composition, the salt upper and lower the laminated constantly dissolution, making the interlayer is still in limbo, when interlayer floating is span to reach enough length, mezzanine collapsed. The collapsed mezzanine is very likely to drop bend or cut the casing, which influences the progress of the cavity and shape of the expansion. The main factors affecting the mezzanine collapsed: the interlayer Strength weakening from the brine soak and the mezzanine floating span.

The study from Liu Yan-hui shows the mudstone interlayer between salt mainly is the clay, mixed with illite and montmorillonite; The mudstone interlayer mainly contain of fine-grained particles; its disintegration along the bedding plane, layered cracking soften, with micro-expansion. Liu Jiang has made the uniaxial and triaxial compression tests, found that under uniaxial compression, elastic modulus and elastic limit descending rock salt, mudstone, inter layer. But Poisson's ratio descending rock salt, mudstone, inter layer. Especially in the three compression tests, you can see the salt rock deformation is significantly larger than the interlayer deformation, showing the deformation uncoordinated. Gao Hong-bo analysis the influence of plaster strength from brine soaked and found that the strength of the gypsum rock has the trend of weakening with temperature increasing, salt concentration increases, the immersion time, the cohesion and internal friction angle of the gypsum rock showed a decreasing trend with the immersion time; To distinguish the failure modes and its differences of the gypsum rock under different conditions, he definite the brittle indicators of the rock deformation failure for damage to the stiffness, approximated by the slope of destruction segment of the rock under uniaxial compression stress-strain curve peak.

The surface subsidence and stability of group cavity: During Salt cavity construction process, due to lack of strength of roof, the chamber volume contraction is too large, the distance spacing between single cavity is too small, the safety pillar collapse, making group cavity instability and ultimately lead to surface subsidence.

The instability of salt cavity mainly contains cavity collapsed, pillar damage and surface subsidence, the worst harmful disasters. Liu Cheng-lun study on thin layer rock salt deposits, it show the stability of the cavity was significantly enhanced with the decrease of the height of cavity, but decreasing with the increase of span and the influence of inclination on the stability of the cavity is very small; the cylindrical cavity is the most stable, followed by an inverted cone; factors affecting the melting chamber stability from strong to weak order as: the height of cavity, the geometric shape of the cavity, the span of cavity, the layer dip of rock salt. Jiang De-yi summarized factors influencing the stability of rock salt cavity such as ground stress, the rock physical and mechanical properties, groundwater, geological structure, thickness of the salt layer), gives the stress distribution law of rock salt cavity, the law of stress release of the cavity roof, instability criterion and stability control method for salt cavity, laying a theoretical foundation for stability control and the feasibility of the strategic petroleum reserve.

\section{THE MEASURES TO AVOID DISASTERS IN CAVITY CONSTRUCTION PERIOD}

The design of cavity shape: The stability of gas storage of salt rock is the key parameter to evaluate the property of cavity of rock salt. At beginning, we need to make out the design of cavity construction which contains the cavity shape design. The cavity with different shape has inconsistent mechanical properties, so was the stability of cavity. We must consider the specific formation occurrence conditions to make out the reasonable cavity shape. The study shows that the ellipsoidal cavity has good stability and mechanical properties and in the design, the ratio between the height and width of the cavity is the key factor affecting the cavity stability which the general value is about 1.5. For its good stability, we choose the inverted pearshaped cavity to avoid being out of shape of the cavity. And the other matter to be attention is the distance between adjacent cavities must not be too close, or the deformity of cavity shape and the surface subsidence will occur.

The choice of washing techniques: The location, shape and its size of the gas storage of rock salt is the ultimate target and basic of washing techniques. The washing techniques include the circulating systems, water flow and the settings of pipes. In the reverse circulation method, the brine is withdraw from the bottom of the cavity which is higher that is help to improve the efficiency of cavity construction, but it increase the difficulty to control the shape of cavity, to keep the stability of cavity, to avoid the collapse and to protect the roof of cavity. In the direct circulation method, the brine is discharged from the upper of the cavity which has low density, low efficiency, but the shape of cavity can be artificially controlled and the cavity is not easy to collapse.

Only to improve the speed of cavity construction, the high flow rate should be better. However the high flow rate will be constrained by the equipment conditions, so in activity, so we must find out the 
optimal working flow. The distance between washing pipes is the key factor which has important influence to the efficiency of cavity construction and the stability of cavity.

The protection to cavity roof: Due to gravity, in the process of cavity construction, the dissolution rate of the upper rock salt in the vertical direction is higher than the horizontal rock salt, if not to control, it will rapidly dissolve into the top of the salt layer, less than the gas storage requirement. The protection to cavity is the key to the control the cavity shape, the main measure we need to do is protect the salt layer upper not to contact with the fresh water. The blanket material generally using oil or injected gas has been added to form the oil pad or gas pad between the brine with the cavity roof. There are two ways to inject blanket material, for the first method, the blanket material is draw into the cavity together with the fresh water from the center tube, in the other way, the blanket material is injected from the annular space between the production cashing with the intermediate tube. The thickness of pad has not uniform standards, in order to facilitate the measurement and control, usually the thickness of air pad is larger than the oil pad. Once the blanket pad is insufficient, it is timely to recharge.

The detection technology: The diameter of large cavity may reach to 100 meters, so it is hard to detect its volume and shape by using conventional logging tools. After period of research and practice, a detect technology of ultrasonic measurements eventually formed after many attempts including the measurement mode of electromagnetic wave and ultrasonic. Using the analysis results of sonar probing, we can make out the finite element simulation, the calculation of the cavity dynamic characteristics, the working pressure range, the closed and creep speed of cavity and so on. At abroad, the sonar detective technology has been successfully applied to the analysis and study of gas storage, the domestic sonar logging technology in this field is just beginning. We must make the periodic detection and evaluation to the volume and shape of the cavity during the construction of cavity.

\section{CONCLUSION}

To statistical analysis the disasters in process of cavern building with multi-interlayer in china, it shows that: taking Chinese geological conditions for example, the disasters during cavern construction in salt beds with multi-interlayer caused the failure of cavity can be divided into three categories: geological conditions in beds; the damage of cavity wall in the process of cavity building and the distortion of cavity shape expansion. To avoid these disasters usually happened during cavity construction, some problems need to be attention has been proposed, including the design of cavity shape, the choice of washing techniques, the protection to cavity roof and the detection technology. It is necessary to choose the inverted pear-shaped cavity which has high stability and good mechanical properties. During the construction of cavity, it is important to choose the washing technology, to protect the roof of cavity and to detect the shape of the cavity.

\section{ACKNOWLEDGMENT}

This study was supported by the Nation Basic Research Program of China (2009CB724606), Chinese Science Foundation (51074198), Chongqing Natural Science Foundation (No.2010BB6044).

\section{REFERENCES}

Ehgartner, B.L. and B.Y. Park, 2009. Sensitivity of storage field performance to geologic and cavern design parameters in salt domes [R]. Technical Report No. SAND 2009-1278, Sandia National Labs., Albuquerque, NM.

Fuenkajorn, K., 2010. Prediction of cavern configurations from subsidence data $[\mathrm{J}]$. Eng. Geol., 110(1-2): 21-29.

Jiang, D., J. Chen, J. Liu, L. Zhou and C. Wang, 2009. Experimental research on acoustic and dissolved properties of stress damaged salt rock [J]. Rock Soil Mech., 30(12): 3569-3573.

Kazemi, H. and F.W. Jessen, 1964. Mechanism of flow and controlled dissolution of salt in solution mining [R]. SPE J., 4(4): 317-328.

Liang, W., S. Xu, J. Mo, D. Wu and C. Zhang, 2010. Test study of strain rate effects on mechanical performances of salt rock [J]. Chin. J. Rock Mech. Eng., 29(01): 43-50.

Tao, Z. and Z. Ma, 2009. The research of salt cavern 3D modeling technology [C]. Proceeding of the Asia-Pacific Conference on Information Processing, APCIP, 2: 241-243. 